\title{
The Holy Grail of energy? A content and thematic analysis of the presentation of nuclear fusion on the Internet
}

\section{Christian Oltra, Ana Delicado, Ana Prades, Sérgio Pereira and Luísa Schmidt}

\begin{abstract}
The Internet is increasingly considered as a legitimate source of information on scientific and technological topics. Lay individuals are increasingly using Internet sources to find information about new technological developments, but scientific communities might have a limited understanding of the nature of this content. In this paper we examine the nature of the content of information about fusion energy on the Internet. By means of a content and thematic analysis of a sample of English-, Spanish and Portuguese-language web documents, we analyze the structural characteristics of the webs, characterize the presentation of nuclear fusion, and study the associations to nuclear fission and the main benefits and risks associated to fusion technologies in the Web. Our findings indicate that the information about fusion on the Internet is produced by a variety of actors (including private users via blogs), that almost half of the sample provided relevant technical information about nuclear fusion, that the majority of the web documents provided a positive portrayal of fusion energy (as a clean, safe and powerful energy technology), and that nuclear fusion was generally presented as a potential solution to world energy problems, as a key scientific challenge and as a superior alternative to nuclear fission. We discuss the results in terms of the role of Internet in science communication.
\end{abstract}

KEYWORDS: Content analysis, Web-based content, Fusion energy

\section{Context}

Scientific and technological content is increasingly transmitted through web sites, including government and educational sites, online magazines, blogs and social media. The Internet is becoming more accessible to more people, is used more often to search for information and is increasingly considered as a legitimate source of information $[1,2]$. Public agencies and scientific and educational organizations are investing resources to provide information to the general public on the Web. But also actors with fewer resources, such as small NGOs or individual citizens, are now able to present information online more easily than ever [3]. In a sense, the Internet is improving the access of individuals and stakeholders to the public debate on technological and scientific issues [4]. 
Fusion energy, the potential commercial production of energy from nuclear fusion processes, is clearly an issue where online environments have the potential to increase public knowledge levels by providing easier access to information. Web-based content is also likely to play a role in audiences' attitudes about nuclear fusion developments. In recent years, the European nuclear fusion research and development community has become more aware of the need to take seriously societal awareness of its activities [5]. Among the various public information and dissemination activities, public bodies such as the European Commission, the ITER Organization and the US Department of Energy have created specific web pages about nuclear fusion and related projects. The Internet has also become a useful medium for other actors to discuss the potential benefits and risks of fusion power. But scientific communities might not be aware of this content.

The academic interest in the web-based information about technological and scientific developments has grown in the last years [4]. As an example, recent studies have investigated the presentation of nanotechnology on the Internet and the social media, considered the new window to the nanoscience [3,6-8]. These studies draw on previous research on the media framing of nanotechnology $[9,10]$ and related literatures interested in the framing and presentation of technologies in printed news articles and stories, given its potential impacts on how the audiences understand these technologies [11]. The analysis of web-based information is not new in the academic literature. A number of research projects have recently investigated, for example, health-related information on the Internet [e.g. 12,13], given its potential influence on patient behavior.

Although there are no studies on the presentation of nuclear fusion on the Internet, the printed media coverage of nuclear fusion has been examined by social research conducted in the context of the EFDA-SERF Programme (see a review in [14 and 15]). The basic assumption is that the media has a potentially important role in shaping lay views about fusion technology. The main conclusion of this research is that the media attention to nuclear fusion has been limited and irregular, that it is still early to observe a clear formation of media frames about nuclear fusion and that framing has tended to be purely technological and neutral; and also, that there are no clear data about how the nuclear brand is associated with fusion energy in the media coverage.

More recently, work carried out in the EFDA-SERF Programme [16] has found a limited but constant over time coverage of nuclear fusion in the main newspapers in the studied countries. Fusion energy is generally shaped in positive terms and framed as a potential source of clean and unlimited energy and as a key solution to energy problems, with very little links to the "nuclear brand" or "stigma" (negative resonances in terms of fear, stigma) (see $[17,18]$ for a general discussion on the nuclear stigma and $[5,19,20]$ for a discussion on the role of the "nuclear brand" in the discourse about nuclear fusion). Little is known about the presentation of fusion technology on the Internet and, specifically, about the role of various metaphors and associations (e.g. "sun's energy", "nuclear brand", "the holy grail"). 


\section{Objective}

The objective of this study was to examine the nature of the web-based information about fusion energy. First, given the limited research on the content of web documents about fusion energy, we sought to describe the structural characteristics of the web sites. Second, we explored how the technology is portrayed on the Internet by examining the nature of the content of the web documents providing information about fusion energy. We specifically examined three questions: How do the web documents link fusion energy to nuclear fission technologies? What types of ideas are employed in the presentation of fusion energy in the web documents? What are the main benefits and costs associated to fusion power on the web-based content?

\section{Method}

We implemented a quantitative and qualitative content analysis with a sample of web documents about nuclear fusion including web pages, blogs and online documents in Spanish, Portuguese and English. We selected these three languages based on the capabilities of the research team. We used Google Search ${ }^{1}$ engine during a one-day period in March 2013 to draw the sample for the study (consistent with other authors' recommendations for sampling Internet-based content. See, e.g. [21]). We initiated Google searches for fusion energy with three key phrases: "fusion energy", "nuclear fusion" and "nuclear fusion advantages and disadvantages" in English and "fusión nuclear", "energía de fusión" and "fusión nuclear ventajas y desventajas" in Spanish, "fusão nuclear", "energia de fusão" and "fusão nuclear, vantagens e desvantagens" in Portuguese. For each search, the first 50 results were collected. We included various sets of data: Web pages (from official and non-official Web sites), blog entries, articles in online magazines and online documents. We discarded scientific papers and Ph.D. thesis. The search procedure resulted in a final sample of 139 web documents in the Spanish-language sample, 106 in the Portuguese sample and 147 in the English-language sample (Table 1). The list of the web documents included in the study is available from the author.

We developed a content analysis protocol for gathering basic quantitative data on various dimensions of the online documents: its structural characteristics, the nature of the content, the risks and benefits associated with fusion power and the general position on fusion (assessed by the researcher). The coding protocol was based on the protocol developed in a previous EFDA-SERF task [16]. Two researchers independently assessed the content of a small subsample of web documents using the content analysis sheet.

The structural characteristics of each web document, including the type of web document, the type of author and whether or not the web page had a picture, were registered in a database. We also registered other general variables, including whether or not fusion was the core subject of the document, whether or not the web document mentioned nuclear fission, the level of information about nuclear fusion included in the web document,

\footnotetext{
${ }^{1}$ Google was chosen as the search engine because it is considered the most popular search engine.
} 


\begin{tabular}{|lccc|}
\hline Google search terms & $\begin{array}{c}\text { Spanish- } \\
\text { language }\end{array}$ & $\begin{array}{c}\text { English- } \\
\text { language }\end{array}$ & $\begin{array}{c}\text { Portuguese- } \\
\text { language }\end{array}$ \\
\hline Fusion energy & 50 & 47 & 41 \\
Nuclear fusion & 48 & 50 & 37 \\
Nuclear fusion advantages & 41 & 50 & 28 \\
and drawbacks & 139 & 147 & 106 \\
\hline
\end{tabular}

Table 1. Final sample (number of web documents collected).

the general position toward fusion and whether or not various benefits and drawbacks of fusion power were mentioned in the information. The list of benefits and drawbacks was built after a careful reading of web-based and printed media content about fusion energy.

In order to complement the quantitative content analysis, we developed a thematic analysis [22, 23] based on a qualitative coding protocol to extract thematic content for further qualitative analysis (Table 2). This involved the development of a template in the form of codes to be applied as a means of organizing the text for subsequent analysis and interpretation. We searched for various key themes in the web-based content about fusion energy; mainly how fusion is introduced in the web documents, how it is described, the general evaluation of fusion, its relationship with nuclear fission, the viability of fusion, and the presentation of the potential benefits and costs of fusion power. These codes were generated by a deductive and inductive approach [24]. We paid special attention to specific statements or messages regarding fusion energy.

\section{Results}

\section{Structural Characteristics of the Web documents}

The first research question examined the structural characteristics of the web sites containing information about fusion. Of our 137 units included in our Spanish-language, we found three main types of web documents: web pages (33\%), articles (28\%) and blog entries (24\%). Only $10 \%$ of the web documents were online documents (factsheets, etc.). Of the 149 units in the sample in English, 65\% were web pages, $21 \%$ articles and only $7 \%$ blog entries. In the Portuguese-language sample, $54 \%$ of the records were web pages, $9 \%$ media articles and $14 \%$ online documents and blog entries. Interestingly, blogs were overrepresented in Spanish.

Regarding the authorship of the web documents, no single type of author is responsible of more than $25 \%$ of the web pages. The web documents found in Spanish $(\mathrm{n}=139)$ are mainly produced by the media $(27 \%)(9 \%$ printed media in electronic version and $18 \%$ electronic media), private users $(24 \%)$ and education organizations (20\%). Official sites and scientific organizations were marginally relevant in producing information about fusion on the Internet (2\% and 5\% respectively). In the English-language documents $(n=147)$, the majority are produced by the media $(25 \%)$ (mainly electronic media), 


\begin{tabular}{|c|c|c|}
\hline $\begin{array}{l}\text { Dimensions } \\
\text { of analysis }\end{array}$ & Main questions to be addressed & Sample statements \\
\hline Context & $\begin{array}{l}\text { How is fusion introduced? What is the } \\
\text { context of the discussion? }\end{array}$ & $\begin{array}{l}\text { "By } 2050 \text {, an expected rise in global } \\
\text { population from six billion to nine bil- } \\
\text { lion and better living standards could } \\
\text { lead to a two to threefold increase in } \\
\text { energy consumption". }\end{array}$ \\
\hline Description & $\begin{array}{l}\text { How is fusion described? What is fu- } \\
\text { sion aimed at? What metaphors are } \\
\text { used? }\end{array}$ & $\begin{array}{l}\text { "Fusion Energy is the energy that pow- } \\
\text { ers the sun". }\end{array}$ \\
\hline Evaluation & $\begin{array}{l}\text { How does the author evaluate fusion? } \\
\text { What kind of claims does the author } \\
\text { provide to support his position? }\end{array}$ & $\begin{array}{l}\text { "We'd like to scale it down to earth. } \\
\text { Fusion is touted as the ultimate energy } \\
\text { supply". }\end{array}$ \\
\hline $\begin{array}{l}\text { Relation to } \\
\text { fission }\end{array}$ & $\begin{array}{l}\text { Is fusion linked to nuclear fission? In } \\
\text { what terms? }\end{array}$ & $\begin{array}{l}\text { "Fusion is very different from fission. } \\
\text { In fission, energy is produced as a re- } \\
\text { sult of splitting apart heavy uranium } \\
\text { atoms to release nuclear energy, the } \\
\text { products of which are radioactive". }\end{array}$ \\
\hline Viability & $\begin{array}{l}\text { What is said about the feasibility of fu- } \\
\text { sion? }\end{array}$ & $\begin{array}{l}\text { "Human-engineered fusion has already } \\
\text { been demonstrated on a small scale." }\end{array}$ \\
\hline Benefits & $\begin{array}{l}\text { What are the benefits of fusion? How } \\
\text { are they characterized? }\end{array}$ & $\begin{array}{l}\text { "A solution to climate change and an } \\
\text { age of clean, cheap energy" }\end{array}$ \\
\hline Costs & $\begin{array}{l}\text { What are the main costs/disadvantages } \\
\text { of fusion according to the author? How } \\
\text { are they discussed? }\end{array}$ & $\begin{array}{l}\text { "At the moment, it is very, very expen- } \\
\text { sive" }\end{array}$ \\
\hline
\end{tabular}

Table 2. Coding protocol for qualitative thematic analysis.

education organizations (24\%), associations (14\%), scientific organizations (11\%) and official sites (8\%). The results are slightly different in high-ranked Google results (first five results in each search), where official sites and scientific organizations are slightly overrepresented (18\% compared to $13 \%$ in the total sample), but not as different as one could expect. Interestingly, in the Portuguese-sample $(n=106)$, the media was less relevant in the production of information about fusion (around 10\% of the documents), whereas education (29\%), private users (18\%) and other types of authors such as scientific experts $(10 \%)$ were more relevant.

\section{The presentation of nuclear fusion and its relationship to nuclear fission}

The review of the content of the sites indicated that fusion energy was the core subject in the majority of the web documents. An $83 \%$ of the web documents in the English and Spanish samples were specifically about fusion energy and only $12 \%$ of the web docu- 
ments covered fusion energy as a subsidiary subject in the context of fission. Only $67 \%$ of the articles in Portuguese were specifically about fusion energy. Regarding the level of information provided, almost half of both the English and Spanish samples (47\%) provided relevant information about fusion energy. This percentage was significantly lower in the Portuguese-language sample (around 20\%). Relevant information included a description of the fusion reaction and the available technologies to replicate fusion on the earth and, in some cases, a discussion of the benefits in terms of energy generation. Around 35\% in both samples provided superficial information about fusion (e.g. "Fusion is the process of fusing together light atoms") and around 15\% did not provide any information or definition about fusion. In the Portuguese-language sample, a higher percentage of web documents did not provide any information (22\%) about fusion or provided very superficial information (50\%). Thus, $15 \%$ of all web documents (mainly articles in printed media, advertisements and web sites of scientific research groups) mentioned nuclear fusion but did not explain the main features of nuclear fusion or provide a wider discussion about potential benefits and risks from fusion energy.

A first qualitative research question was related to how fusion energy is introduced or presented in the web. We found two key frames used as a contextualization of fusion: "fusion power as a solution to world energy problems" and "fusion as a scientific and technological challenge". Linked to the energy problem frame, two main ideas are discussed: the growing world energy demand and climate change. For example, a blog entry introduced nuclear fusion in the following terms: "As we face the dangers of increased greenhouse gases in the atmosphere and pass peak oil production, fusion becomes a very attractive option for supplying this future demand" (doc. 2.19). There seems to be an agreement in the Web-based information about fusion power that new energy sources must be developed to face energy and environmental problems. The underlying message in the majority of the web documents is represented in the following excerpt: "We need to innovate alternative energy sources now more than ever ... and our choices are limited" (doc. 2.11)

Many other news articles from online magazines and websites from scientific or educational organizations simply introduce nuclear fusion as a technological and scientific challenge. In this context, the energy problem has a secondary role. Fusion is presented as a scientific problem that is being investigated around the World. A web document, for example, states: "It (Cadarache, where ITER, the International Thermonuclear Experimental Reactor, is being built) is the place where 34 nations representing more than half the world's population have joined forces in the biggest scientific collaboration on the planet - only the International Space Station is bigger" (doc. 1.4). We found other less frequent thematic contexts in which fusion energy is introduced such as introductions to nuclear science, the hydrogen bomb, rocket science or cosmology.

The description of nuclear fusion is obviously very similar among the web documents. But there are slight differences in the words emphasized by web documents to define nuclear fusion. The most mentioned words were the following (ordered by frequency): nuclei, sun, process, atoms, hydrogen, reaction, stars, fission, mass, future, bomb, helium, clean. We found three main types of definitions of nuclear fusion. The most frequent 
definition states something similar to: "Fusion produces energy when atoms combine". A second common definition links fusion to the process that happens in the Sun. This metaphor was found frequently found in the Web. For example, one web page states: "Fusion is the process which powers the sun and the stars". Finally, other web documents define nuclear fusion by emphasizing the differences between nuclear fusion and nuclear fission. For example: "In contrast, fission used in today's nuclear reactors, in which atoms are split, generates both energy and radioactive byproducts".

An important research question dealt with the role of associations to nuclear fission in web-based information about nuclear fusion. The data from the content analysis showed that a majority of the web documents in Spanish and English (around 60\%) mentioned nuclear fission, whereas only $35 \%$ of the web documents in the Portuguese-language sample mentioned fission. Web documents collected by searching the keywords "fusion energy" and "nuclear fusion" mentioned nuclear fission less frequently (around 50\% of the cases) than those documents obtained in the search for "nuclear fusion advantages and disadvantages", where $80 \%$ of the sample mentioned nuclear fission. This is due to the fact that a number of the web documents collected by the search for "nuclear fusion advantages and disadvantages" consisted of comparisons between nuclear fusion and fission.

The qualitative analysis of the textual units linking both types of nuclear processes showed the existence of three main messages. First, we registered a set of messages with the following idea: "fusion has many advantages over current fission technologies". This seems to be the most relevant idea when comparing fusion and fission. Nuclear fusion is presented as an alternative to nuclear fission, systematically linked to various risks and disadvantages (mainly related to the environmental and health impacts and to safety). Nuclear fusion can overcome the limitations of fission technology, these web documents report. For example, a web document states: "Fission and fusion can claim many of these same advantages, but fission comes with some serious and all too apparent risks" (doc. 1.21)

First, fusion power is characterized as a cleaner source. For example, a web document in the English-language sample states: "Fusion is a "cleaner" process than fission. i.e. less radioactive by-products" (doc. 3.9). Another relevant set of messages state that fusion is safer than fission. Some web documents argue that accidents such as Chernobyl, Three Mile Island or Fukushima could have not happened in a fusion power plant. Nuclear fusion is characterized as intrinsically safe, while fission technology is portrayed as posing serious risks to safety. Two web documents, for example, state: "Unlike nuclear fission, there is no potential for meltdown" (doc. 2.16), and "unlike fission, nuclear fusion should be safe" (doc. 3.31). Another relevant idea in the web documents is that fusion reactions can produce higher amounts of energy than fission reactions. This message seems to be more relevant in the Spanish-language sample. Finally, some web documents simply emphasize that fusion and fission are two different ways to obtain nuclear energy. In this message, nuclear fission is not necessarily portrayed in negative terms. 


\section{Potential benefits and limitations of fusion power}

An important research question dealt with the number of positive and negative statements about fusion energy mentioned in the Web. We found that a majority (around 75\%) of the web documents studied had one or more positive statements about fusion energy (e.g. fusion is clean, resources are unlimited, etc.). The average number of positive statements was 3.2 per web document. The number of positive statements was slightly higher in the Spanish-language sample (Mean $=3)$ than in the English-language sample $(M=2.4)$. The number of positive statements was higher in the Portuguese-language sample $(\mathrm{M}=4.3)$. Regarding the negative statements, we found that $52 \%$ of the web documents in the sample had one or more negative statements about fusion energy. The average number of negative statements per document was 0.8 for the Spanish-language sample, 0.5 for the Englishlanguage sample and 1.6 for the Portuguese sample.

As can be seen in Ttable 3, the statements most frequently associated with nuclear fusion were related to the nature of the fuel for fusion power, the environmental impacts of fusion, its safety and the capacity for generating large amounts of energy. A large number of the web documents (43\%) emphasized the abundance of resources for nuclear fusion. This idea is linked to the problem formulated in most web documents when introducing fusion: the energy demand will grow in the coming years. A web document, for example, states: "Fuel supplies will therefore last for millions of years". The other two most frequent positive statements about fusion were related to the environmental impacts of fusion energy. Fusion power is generally portrayed as a clean source of energy ( $45 \%$ of web documents). For example, one web page states that "As you know, fusion power is a safe, clean, and sustainable energy source". The idea of non production of radioactive waste was also relevant in the characterization of fusion power (mentioned in $36 \%$ of the sample), and particularly in the Spanish sample, where this feature of fusion was discussed extensively.

Other positive messages included "fusion is safe", "fusion has capacity for generating large amounts of energy" or "fusion is climate neutral". The safety of nuclear fusion was mentioned as a benefit in $37 \%$ of the web documents. Nuclear fusion is generally characterized as being intrinsically safe. These web documents argue that nuclear accidents will not have a place in future fusion reactors, as runaway nuclear reactions are considered impossible in fusion reactors. For example, a web document states: "From a safety standpoint, it poses no risk of a runaway nuclear reaction - it is so difficult to get the fusion reaction going in the first place that it can be quickly stopped by eliminating the injection of fuel" (doc. 1.8).

The climate neutrality of fusion power, the presentation of fusion as an "alternative to nuclear fission" and the idea that "there are successful projects" were mentioned in around $20 \%$ of the web documents (with some significant differences among the samples). The "cost-effectiveness of fusion", the idea of "fusion as an alternative to energy technologies based on fossil fuels", the "benefits for the economy", the "benefits for national energy security", the "promotion of international collaboration" or the potential "contribution of 


\begin{tabular}{|c|c|c|c|}
\hline Key positive statements & $\begin{array}{c}\% \text { of the web } \\
\text { documents }\end{array}$ & Key negative statements & $\%$ \\
\hline $\begin{array}{l}\text { Zero/low environmental impact } \\
\text { (clean) }\end{array}$ & 45 & Technology not ready (unproven) & 37 \\
\hline $\begin{array}{l}\text { Abundant or unlimited resources, } \\
\text { fuel }\end{array}$ & 43 & $\begin{array}{l}\text { Consumes more energy than it } \\
\text { produces }\end{array}$ & 23 \\
\hline Unlimited production of energy & 43 & High costs of investment & 10 \\
\hline Fusion is safe (general) & 37 & (Too) far in the future & 10 \\
\hline $\begin{array}{l}\text { Low/No production of radioactive } \\
\text { waste }\end{array}$ & 36 & $\begin{array}{l}\text { Not clean (production of radioac- } \\
\text { tive wastes) }\end{array}$ & 5 \\
\hline $\begin{array}{l}\text { Climate neutrality (no CO2 emis- } \\
\text { sions) }\end{array}$ & 26 & Human health risks (cancer, etc.) & 1 \\
\hline Alternative for nuclear fission & 18 & Risks to the environment & 1 \\
\hline $\begin{array}{l}\text { Alternative to energy technologies } \\
\text { based on fossil fuels }\end{array}$ & 15 & $\begin{array}{l}\text { There are better options to tackle } \\
\text { energy problems }\end{array}$ & 1 \\
\hline $\begin{array}{l}\text { Cost-effectiveness of energy pro- } \\
\text { duction (efficiency) }\end{array}$ & 12 & $\begin{array}{l}\text { Threat for investments in renew- } \\
\text { able energy/energy efficiency }\end{array}$ & 1 \\
\hline There are successful projects & 12 & Based on non renewable resources & 1 \\
\hline $\begin{array}{l}\text { Answer to growing energy de- } \\
\text { mand }\end{array}$ & 8 & Unsafe (general) & 1 \\
\hline It will be ready in the near future & 5 & $\begin{array}{l}\text { Not a real solution to climate } \\
\text { change }\end{array}$ & 1 \\
\hline $\begin{array}{l}\text { Good for energy security (e.g. en- } \\
\text { ergy independency) }\end{array}$ & 5 & $\begin{array}{l}\text { Government support needed (de- } \\
\text { pendent on subsidies) }\end{array}$ & 1 \\
\hline $\begin{array}{l}\text { Benefits for the economy (invest- } \\
\text { ments, jobs) }\end{array}$ & 4 & $\begin{array}{l}\text { Centralized form of energy pro- } \\
\text { duction }\end{array}$ & 1 \\
\hline $\begin{array}{l}\text { Promotes international collabora- } \\
\text { tion }\end{array}$ & 4 & Form of nuclear energy & 1 \\
\hline $\begin{array}{l}\text { Part of mixed energy supply port- } \\
\text { folio }\end{array}$ & 3 & Uncertain public acceptance & 1 \\
\hline
\end{tabular}

Table 3. Key positive and negative statements on fusion energy in the Internet.

fusion to the mixed energy portfolio" were also mentioned in the sample (in less than $10 \%$ of the web documents).

We also found information regarding the potential risks and drawback of fusion power on the Internet, mainly related to the viability and feasibility of fusion. The most frequently mentioned message, found in more than $37 \%$ of the web documents (specifically, in $22 \%$ of the English-language sample, $33 \%$ of the Spanish-language sample and $65 \%$ of the Portuguese-language sample) was that "fusion technology is not ready" or that "nuclear fusion is a not proven technology". Around $23 \%$ of the web documents, again with 
significant differences among samples, also mentioned that fusion power "consumes more energy than it produces" and that fusion "is too far in the future". Some web documents clearly argued that fusion power will not contribute to the energy system in the coming years. But, interestingly, very few of the web documents discussed the long term frame of fusion energy in totally negative terms.

The costs of the fusion program and of future fusion facilities were mentioned as an important drawback of fusion in around $10 \%$ of the web documents. For example, an educational web site states: "At the moment, it is very, very expensive" (doc. 1.93). Some others discuss the costs of ITER. For example, this web document in the Spanish sample argues that: "The road is winding and expensive. The partners of ITER have approved the budget for the next ten years, and it multiplies by a number of three what was predicted in 2006" (doc. 2.13). Web documents also mentioned the costs of construction and operation of future fusion facilities. Two web pages, for example, state: "fusion reactor needs to be larger and therefore more costly" and "they have to find ways to reduce the costs of construction and operation so that the energy does not become too expensive". Finally, a minor number of web documents (around $4 \%$ of the sample) portrayed fusion power as a dirty source of energy. A Spanish web document, for example, stated: "It is false that nuclear power will not produce radioactive waste"

\section{Is fusion energy viable?}

Beyond the potential benefits and costs of fusion power, the viability and feasibility of fusion energy was another key dimension in the presentation of fusion energy in the Web. We found a variety of elements in the discussion, and two main perspectives: an optimistic view of fusion viability and a negative view of it.

Web documents that presented a negative view of the potential feasibility of fusion provided a main message: "it is highly unlikely that fusion reactor technology will be available in the future". For example, an electronic media article argues: "Then again, claims that net fusion power is just around the corner have been made for decades" (doc. 1.24). Another web document states: "Major questions remain about pulling off this long-dreamed-of technological feat" (doc. 1.41). The questioning of fusion energy viability is built on three main ideas: i) "There are unsolvable technical challenges to fusion power"; ii) "Fusion energy is economically unviable"; and iii) "Fusion reactions cannot create net positive energy". Web documents questioning the future viability of fusion power tend to point to the important technological and scientific challenges facing controlled nuclear fusion. A web document about fusion energy, for example, states: "Scientists have to overcome engineering challenges they face in construction of the reactor and in operating it with a high reliability and availability" (doc. 1.45).

Another web document outlines the difficulties of making fusion power commercially available and states: "I think it's safe to use our fusion quest as the definition of hard. It's a much larger challenge than sending men to the Moon" (doc. 3.20). The idea that fusion energy will never become cheap enough to become commercially viable is also presented 
in some of the web documents. For example, one web page states: "And last, but not least - they have to find ways to reduce the costs of construction and operation so that the energy does not become too expensive" (doc. 1.45). Finally, other web documents also point to the energy balance of fusion power. For example, one web document arguing about the challenges facing fusion energy states: "The fundamental problem is that it takes more energy to run fusion than what fusion can deliver".

But, generally, web documents tend to provide a positive view of nuclear power's viability. This view is represented by the following excerpts from three web documents: "We're getting close!"; "Here are a number of major projects under development that may bring research to the point where fusion power can be commercialized"; "Science may only be a few years away from showing that fusion can work". This optimistic view of fusion energy is based on three main ideas. First, that "nuclear fusion has already been achieved". For example, a web document states: "It is not easy to build a device that runs at ten times the temperature of the Sun, but it is possible... In fact, the European experimental facility, JET - hosted in the U.K., has already done it" (doc. 2.11). Another web page, for example, states "fusion is a routine now". Second, we found the following idea: "fusion power is viable but at a high cost". It is argued that controlled nuclear fusion reactions are achievable, but expensive. For example, a web document states: "As for fusion, the bottom line is not whether we can do it but whether we can do it at a price people will be prepared to pay" (doc. 2.26). Finally, there is the idea that fusion energy has made important progress in the last years. For example, one web document states: "Nevertheless, fusion energy has made significant progress over the last few decades and is now considered as a credible option for clean energy" (doc. 2.18). Fusion is credible, as scientists are making progress. This is the main message.

\section{The portrayal of fusion power}

Finally, a key research question in our study examined whether web documents portrayed a general positive, neutral, negative or an ambivalent view of fusion energy. According to our data, web-based information about nuclear fusion is mainly positive. The results are very similar in the English and Spanish subsamples. In the Spanish-language sample, a 53\% of the web documents were categorized as positive about fusion; $34 \%$ had a neutral presentation of fusion; $9 \%$ were considered ambivalent and $4 \%$ of documents were negative about fusion. Similar results were found in the English-language sample, where $56 \%$ of the web documents were coded as positive, 33\% as neutral, $9 \%$ as ambivalent and $1 \%$ as negative. In the Portuguese-language sample we found a higher percentage of web documents coded as positive (70\%) but also as negative (6\%). Only $22 \%$ of the Portuguese web pages were considered as having a neutral evaluation towards fusion and $2 \%$ as being ambivalent.

An analysis by type of author (Table 4) found that web documents from official sources had the highest rate of positive valuations (93\%), followed by companies (72\%) and scientific organizations $(70 \%)$. Web documents from printed media had also substantially 


\begin{tabular}{|lllll|}
\hline Type of author & $\begin{array}{c}\text { Positive } \\
\%(\mathrm{n})\end{array}$ & $\begin{array}{l}\text { Neutral } \\
\%(\mathrm{n})\end{array}$ & $\begin{array}{l}\text { Negative } \\
\%(\mathrm{n})\end{array}$ & $\begin{array}{l}\text { Ambivalent } \\
\%(\mathrm{n})\end{array}$ \\
\hline Official & $93(13)$ & $7(1)$ & 0 & 0 \\
Electronic media & $54(36)$ & $31(21)$ & $1(1)$ & $13(9)$ \\
Printed media (online version) & $60(12)$ & $30(6)$ & 0 & $10(2)$ \\
Scientific organization & $71(25)$ & $29(10)$ & 0 & 0 \\
Educational & $44(28)$ & $44(28)$ & $3(2)$ & $8(5)$ \\
Associations and NGOS & $52(24)$ & $30(14)$ & $7(3)$ & $11(5)$ \\
Company & $72(8)$ & $27(3)$ & 0 & 0 \\
Scientific expert & $63(12)$ & $31(6)$ & $5(1)$ & 0 \\
Private user (Blog) & $61(41)$ & $27(18)$ & $4(3)$ & $7(5)$ \\
Total & 58 & 30 & 3 & 9 \\
\hline
\end{tabular}

Table 4. General evaluation of fusion power by type of actor.

positive information about fusion (60\%). Educational web sites, private users (blogs) and electronic media had the highest rate of neutral information $(44 \%, 27 \%$ and $31 \%$ respectively). Electronic media, associations and blogs had the highest rate of ambivalent information $(13 \%, 11 \%$ and $7 \%$ respectively). Web documents with a negative position on fusion were mainly authored by environmental NGOs and associations and blogs linked to educational web sites.

In general, positive web documents about fusion (around 58\% of the sample) emphasized a variety of benefits and positive features of nuclear fusion. The main characteristic of these web documents was the framing of fusion as a positive development (even referring to the Holy Grail of energy), due to the potential contribution of fusion power as a solution to energy problems, its advantages compared to other energy options or its scientific character. We found four main positive messages about fusion power in web documents with a positive attitude towards fusion. The first one states that "fusion energy is the key solution to world energy problems". Fusion energy is considered the hope for the future of energy. The three following excerpts exemplify this view:

"Fusion energy promises to be the final solution to the energy problems of the planet. Similar to the operation of stars such as the sun, it will generate clean energy in large quantities at very low cost" (doc. 1.35)

"Nuclear fusion is the 'perfect energy source'" (doc. 1.27)

"It's the Holy Grail, it's the pot of gold at the end of the energy rainbow" (doc. 1.5)

The second message states that "fusion energy has substantial benefits, namely, that is clean, safe and reliable and it is worthwhile to research it". Nuclear fusion is depicted here as a clean, unlimited and safe source of energy and as having fundamental benefits in 
important areas for societies. A third positive message compares fusion energy to nuclear fission and other conventional (e.g. fossil fuels) and renewable sources of energy. This message presents the idea that "fusion energy is a better option than fission energy or fossil fuels". Another group of positive messages about fusion energy consider nuclear fusion "as a key technological and scientific challenge for societies". Finally, less relevant positive messages framed the development of nuclear fusion as a critical issue for the nation.

Neutral web documents (accounting for around 30\% of the entire sample) tended to provide technical definitions of nuclear fusion, discuss the technological challenges facing fusion developments, introduce the different ways of producing fusion reactions and present the developments in fusion research. Neutral web pages mentioned very few potential benefits and drawbacks of fusion energy, did not discuss the viability of fusion energy in negative terms, did not provide an energy policy context for discussing fusion and or stated the advantages of nuclear fusion over other energy sources. Nuclear fusion was introduced as a scientific and technological issue with very few positive impacts or controversies associated with it.

We found very few negative web documents about fusion energy $(n=13)$ in our study. In the Spanish sample $(n=6)$, two of them were authored by environmental NGOs, three by private users via blog entries and one by a research scientist. The key messages in these web documents were the following: "we do not need fusion energy", "the idea of fusion as an unlimited source of energy is not true" and "fusion energy is not viable". The information provided in these documents rejected the potential benefits of fusion. The arguments behind these messages were diverse, but mainly referred to the following ideas: fusion energy is not clean and not safe; fusion power is not viable; fusion energy is not a solution to climate change; fusion resources are not unlimited; and the development of fusion power would have negative societal and political impacts. In the English-language sample, only one web document was coded as mainly negative about fusion energy. This does not mean that the costs and drawbacks of fusion energy were not discussed in web documents coded into other categories.

Finally, ambivalent web documents (9\%) were considered those combining positive and negative feelings towards fusion energy. We considered as ambivalent those web documents providing messages such as "Fusion power offers the prospect of an almost inexhaustible source of energy for future generations, but it also presents so far insurmountable scientific and engineering challenges". But also, those web documents showing a skeptical or cynical attitude towards nuclear fusion, such as the one represented in the following excerpt: "The energy form has been touted as having the potential to provide almost limitless supplies of clean, safe and sustainable energy without the downsides of nuclear power produced by fission" (doc. 1.40). Generally, ambivalent documents portrayed fusion energy as having many potential benefits, but also unsolvable challenges. We categorized around $10 \%$ of the web documents in the total sample as having an ambivalent position towards fusion energy. The results were, interestingly, very similar in the Spanish- and the English-language sample. 


\section{Conclusions}

A large amount of information about nuclear fusion is easily available from the Internet for individuals in the form of web pages, online documents, blogs, forums and videos. Compared to traditional media such as newspapers, the Internet offers an easier access to information about technologies, but also allows a wider variety of actors to discuss their potential benefits and risks. This study was designed to examine the characteristics and content of web documents providing information about fusion energy and to explore the ways in which fusion energy is presented to the general public.

The examination of the web documents indicated that the majority of web documents about fusion energy (especially in the English and Spanish-language samples) provided relevant and contextual information about this future energy technology to potential interested users. We also found that the information about fusion energy on the Internet is produced by a variety of actors, from electronic media to scientific organizations or private users. The analysis of the content showed that the information provided about fusion was mainly positive and that the web documents contained large numbers of messages underlying the potential benefits of fusion power, mainly those related to the existence of unlimited resources, the environmental impact and safety. Fusion power was generally presented as a solution to the energy and environmental challenges of future society, a key technological and scientific challenge and a superior form of nuclear energy. The portrayal of fusion as an unproven technology also played a role in web-based information about fusion. But, interestingly, the long term frame of fusion power was mentioned by very few web pages in our sample.

Although we could not establish a systematic comparison with previous printed media studies [16, 25], we find some differences between web-based content and print newspapers' content. First, web documents, compared to the majority of printed media articles, provided a deeper and more diverse contextualization of fusion. The majority of the web documents in our sample was specifically about fusion energy and provided enough information about fusion energy to generate some level of understanding about the technology among readers, whereas most of the printed news articles did not provide relevant information about fusion or were even marginally related to fusion. Fusion is shaped as a new technology producing clean and endless energy and as a potential solution to energy problems. Both the web-based content and the printed media content provided, on average, a positive picture of nuclear fusion, portraying it as a new technology to generate clean and endless energy and as a potential solution to world energy problems and discussed the same themes (environmental impacts, abundant or unlimited resources, technologic feasibility, etc). The main qualitative difference between web-based content and print newspapers' content was that in the web-based content we found a richer, in terms of variety and depth, set of messages, metaphors and descriptions about fusion energy.

Regarding the role of the associations to nuclear fission in the web-based presentation of fusion energy, we found no evidence of a "nuclear brand" linked to fusion. Although nuclear fission was frequently mentioned in the web documents, it was mainly mentioned 
to emphasize the advantages of nuclear fusion over fission technologies, such as a greater safety factor and a greater electricity generation capacity. Some web documents, for example, emphasized the message that accidents such as Chernobyl, Three Mile Island or Fukushima would not happen in a future fusion power plant. In a sense, the role of the nuclear label or "brand" seems to be very limited in the presentation of fusion power on the Internet. Fusion is generally portrayed as an alternative and a better option than fission technologies and it is not generally associated with the risks and drawbacks traditionally associated with nuclear energy. This does not necessarily coincide with the role it could play, under certain circumstances, in the lay reasoning about fusion technology [21].

We acknowledge some limitations of this study. First, there is the question of how individuals will make use of web-content about fusion energy. There is some evidence suggesting that a typical user of Google Search will likely click only the first three results [26]. But, on the other hand, other studies suggest that individuals seeking for deeper information will likely spend at least 30 minutes on a search and visit two to five different web sites [1]. There is also the question of the type of people who will likely access web-based content about scientific and technological issues, as well as the kind of impact that this content may have on their attitudes and levels of understanding about these topics (see [4] for a discussion on this issue). Other limitations are related to the fluid nature of the Web, as some of the web documents analyzed in this article may no longer be active, and to the fact that Google delivers different results for different users. We gathered the 50 records in each search, and compared the results from three different computers, so this should not have affected the results of the study. Another limitation of the study is that there may have been observer bias in determining the general position of web documents on fusion energy. We developed a common criterion among coders, but this could have been insufficient. Finally, we did not address the quality and accuracy of information nor whether the quality was provided by trustworthy sources.

It is important to acknowledge the potential of the Internet as a new context for public engagement with scientific and technological developments such as fusion, as well as its potential effects on public attitudes. The Internet is increasingly considered as a legitimate source of information on scientific and technological topics [2] and it is helping a wider variety of online users to gain comparatively more knowledge about science and technology [6]. The Internet has generated new opportunities for citizen learning about scientific and technological issues, expanded the breadth and channels of science communication and provided new opportunities for stakeholders to influence the message [4]. The Internet has already become part of science communication, but there is no systematic understanding of how such media contributes to the debates about science [27]. In this sense, the role of the Internet, and especially its 2.0 version, on science communication has become a significant research question [2]. For instance, as indicated by Peters et al. [28], not all forms of online science communication are conceptually different from traditional media. In our research, we gathered web-based records from blogs and wikis, but also from traditional media and institutional websites. In a sense, our research suggests that the Internet might have the effect of widening, as compared to traditional media, 
the variety of messages and information available to the public on scientific issues, as well as the variety of actors involved in the production of this information. But this is a question that deserves more systematic research. This paper has aimed at contributing to the empirical literature on the Internet's presentation of technological and scientific developments, but also on the role of the Internet on science communication.

\section{Acknowledgments}

This work has been carried out within the framework of the EUROfusion Consortium and has received funding from the European Union's Horizon 2020 research and innovation programme under grant agreement number 633053. The views and opinions expressed herein do not necessarily reflect those of the European Commission.

\section{References}

[1] S. Fox and L. Rainie (2002), Vital Decisions: How Internet Users Decide What Information to Trust When They, Pew Internet \& American Life Project, Washington, DC, U.S.A. .

[2] D. Brossard (2013), "New media landscapes and the science information consumer", Proceedings of the National Academy of Sciences 110(Supplement 3): 14096-14101.

[3] J. Gerhards and M.S. Schäfer (2010), "Is the internet a better public sphere? Comparing old and new media in the USA and Germany", New Media \& Society 12(1): 143-160.

[4] S. Krimsky (2007), "Risk communication in the internet age: The rise of disorganized skepticism", Environmental hazards 7(2): 157-164.

[5] A.P. López, T. Horlick-Jones, C. Oltra and R. Solá (2008), "Lay perceptions of nuclear fusion: multiple modes of understanding", Science and public policy 35(2): 95-105.

[6] M.A. Cacciatore, A.A. Anderson, D.H. Choi, D. Brossard, D.A. Scheufele, X. Liang and A. Dudo (2012), "Coverage of emerging technologies: A comparison between print and online media", New Media \& Society 14(6): 1039-1059.

[7] X. Liang, A.A. Anderson, D.A. Scheufele, D. Brossard and M.A. Xenos (2012), "Information snapshots: What Google searches really tell us about emerging technologies", Nano Today 7(2): 72-75.

[8] K.K. Runge, S.K. Yeo, M. Cacciatore, D.A. Scheufele, D. Brossard, M. Xenos and L.Y.F Su (2013), "Tweeting nano: How public discourses about nanotechnology develop in social media environments", J. Nanopart. Res. 15(1): 1-11.

[9] M.D. Cobb (2005), "Framing effects on public opinion about nanotechnology", Sci. Comm. 27(2): 221-239.

[10] C. Wilkinson, S. Allan, A. Anderson and A. Petersen (2007), "From uncertainty to risk?: Scientific and news media portrayals of nanoparticle safety", Health, Risk \& Society 9(2): 145-157.

[11] V. Price, D. Tewksbury and E. Powers (1997), "Switching Trains of Thought The Impact of News Frames on Readers' Cognitive Responses”, Commun. Res. 24(5): 481-506.

[12] M.K. Lapinski (2006), "StarvingforPerfect. com: a theoretically based content analysis of pro eating disorder Web sites", Health Communication 20(3): 243-253.

[13] M. Chung, R.P. Oden, B.L. Joyner, A. Sims and R.Y. Moon (2012), "Safe Infant Sleep Recommendations on the Internet: Let's Google It”, The Journal of pediatrics 161(6): 1080-1084. 
[14] A. Prades López, T. Horlick-Jones, C. Oltra and R. Solá (2007), Social Perception of Large Research \& Development Programs: A review of Social Science Research, Colección Documentos Ciemat, Ciemat, Madrid, Spain.

[15] EFDA (2006), European Fusion Development Agreement Socio-Economic Research in Fusion (SERF, 1997 - 2005). Short version of the 2005 Status Report, EFDA.

[16] L. Schmidt, A. Horta, S. Pereira and C. Oliveira (2013), Comparative Analysis of the public discourse about fusion and nuclear energy before and after Fukushima, Internal report for the EFDA-SERF Programme.

[17] R. Gregory, J. Flynn and P. Slovic (1995), “Technological Stigma”, American Scientist 83: 220-223.

[18] R. Kasperson, N. Jhaveri and JX. Kasperson (2001), "Stigma and the Social Amplification of Risk: Toward a Framework of Analysis", J. Flynn, P. Slovic and H. Kunreuther eds., Risk, Media and Stigma, Earthscan, London, U.K. .

[19] O. Renn (1998), “The methodological challenge”, presentation at EFDA-SERF workshop, 9-10 February, Madrid, Spain.

[20] T. Horlick-Jones, A. Prades and J. Espluga (2012), "Investigating the degree of "stigma" associated with nuclear energy technologies: A cross-cultural examination of the case of fusion power", Pub. Underst. Sci. 21(5): 514-533.

[21] S.J. McMillan (2000), "The microscope and the moving target: The challenge of applying content analysis to the World Wide Web", Journalism and Mass Communication Quarterly 77: 80-98.

[22] R.E. Boyatzis (1998), Transforming qualitative information: Thematic analysis and code development, Sage.

[23] V. Braun and V. Clarke (2006), "Using thematic analysis in psychology", Qualitative research in psychology 3(2): 77-101.

[24] A.A. Coffey and P. Atkinson (1996), Making sense of qualitative data: Complementary research strategies, Sage.

[25] C. Oltra, P. Román and A. Prades (2013), "Media Coverage of Nuclear Energy after Fukushima”, Informes Técnicos Ciemat, Ciemat.

[26] Optify (2010) The Changing Face of SERPs: Organic Click Through Rate, report available at: http://www.my.epokhe.com/wp-content/uploads/2011/05/Changing-Face-of-SERPS-OrganicCTR.pdf.

[27] I. Kouper (2010), "Science blogs and public engagement with science: practices, challenges, and opportunities”, JCOM 9(1): A02.

[28] H.P. Peters, S. Dunwoody, J. Allgaier, Y.Y. Lo, and D. Brossard (2014), "Public communication of science 2.0", EMBO reports 15(7): 749-753.

\section{Authors}

Christian Oltra is a researcher at the Socio-technical Research Centre of CIEMAT, Spain. His research interests include risk perception, environmental behavior and the social acceptance of new technologies. E-mail: christian.oltra@ciemat.es

Ana Delicado is a research fellow at the Institute of Social Sciences, University of Lisbon, Portugal (ICS-UL). Her research has been mainly concerned with the social studies of science. E-mail: ana.delicado@ics.ul.pt 
Ana Prades is the head of the Sociotechnical Research Centre at CIEMAT. Her research has mainly focused on Risk Perception and Communication, as well as on Public Involvement on environmental and energy related matters. E-mail: ana.prades@ ciemat.es

Luisa Schmidt is a senior researcher at the Institute of Social Sciences, University of Lisbon, Portugal (ICS-UL). She currently leads the ICS Research Line on Sustainability: Environment, Risk and Spaces. Her research has been mainly concerned with the sociology of the media and environmental sociology studies. E-mail: mlschmidt@ics.ul.pt

Sérgio Pereira. Graduated in Sociology at the Instituto de Ciências do Trabalho e da Empresa (ISCTE) in 2004. Member of research team of Observa - Ambiente Sociedade e Opinião Pública - Instituto de Ciências Sociais, since 2011.

E-mail: sergio.rpereira@gmail.com

How to Cite: C. Oltra, A. Delicado, A. Prades, S. Pereira and L. Schmidt, "The Holy Grail of energy? A content and thematic analysis of the presentation of nuclear fusion on the Internet", JCOM 13(04)(2014)A01. 